\title{
Risk-scoring methods for prediction of postoperative stroke after coronary artery bypass surgery
}

Olli-Pekka Kangasniemi, MS, Johannes Luukkonen, MS, Fausto Biancari, MD, PhD, Enrico Leo, MD, Sailaritta Vuorisalo, MD, PhD, Risto Pokela, MD, PhD, and Tatu Juvonen, MD, PhD, Oulu, Finland

T wo simple risk-scoring methods for prediction of stroke after coronary artery bypass grafting (CABG) have been derived by the Northern New England Cardiovascular Disease Study Group (NNECVDSG) ${ }^{1}$ and the Multicenter Study of Perioperative Ischemia (McSPI) Research Group. $^{2}$ We have evaluated them in a series of patients who underwent isolated on-pump CABG.

\section{Clinical Summary}

From January 1992 to December 1993, 1098 patients undergoing isolated on-pump CABG were considered for prospective studies evaluating antibiotic prophylaxis strategies. ${ }^{3}$ The records of these patients were retrospectively reviewed to retrieve the variables included in the NNECVDSG and McSPI stroke risk-scoring methods, and we were able to calculate the above-mentioned scores in 928 patients (Table 1). Causes of late death have been obtained from a national registry (Tilastokeskus).

Statistical analysis was performed with SPSS statistical software (version 10.0.5; SPSS Inc, Chicago, Ill). The Fisher exact test and the Mann-Whitney test were used for univariate analysis. Receiver operating characteristics (ROC) curves were used for identification of the best cutoff value of continuous variables in predicting postoperative adverse outcome as the value with the best sensitivity and specificity. The Kaplan-Meier method was used to estimate the long-term survival, and Cox regression was used to evaluate the effect of variables on the long-term outcome. Logistic regression and Cox regression with the help of backward selection were used for multivariable analysis. Only preoperative variables whose $P$ values were $<.05$ on univariate analysis were considered for inclusion in the regression model. During the inhospital stay, 17 patients $(1.8 \%)$ had a stroke, $3(0.3 \%)$ had transient ischemic attacks (TIAs), 309 (33.3\%) had atrial fibrillation, $19(2.0 \%)$ had pneumonia, $2(0.2 \%)$ had renal failure, and 4 $(0.4 \%)$ had low cardiac output syndrome. Five patients $(0.5 \%)$ died during the immediate postoperative period, 2 of them having had a postoperative stroke.

From the Division of Cardio-thoracic and Vascular Surgery, Department of Surgery, Oulu University Hospital, Oulu, Finland.

Received for publication Aug 19, 2005; revisions received Sept 12, 2005; accepted for publication Sept 28, 2005.

Address for reprints: Fausto Biancari, MD, PhD, Division of Cardiothoracic and Vascular Surgery, Department of Surgery, Oulu University Hospital, PO Box 21, 90029 Oulu, Finland (E-mail: faustobiancari@ yahoo.it).

J Thorac Cardiovasc Surg 2006;131:734-5

$0022-5223 / \$ 32.00$

Copyright $\odot 2006$ by The American Association for Thoracic Surgery doi:10.1016/j.jtcvs.2005.09.059

\section{TABLE 1. Preoperative risk factors}

\begin{tabular}{lc}
\hline Variable & No. (\%) \\
\hline Age (y) & $60.4 \pm 8.4$ \\
Women/men & $722(77.8) / 206(22)$ \\
Body mass index $\left(\mathrm{kg} / \mathrm{m}^{2}\right)$ & $27.9 \pm 3.6$ \\
Chronic obstructive pulmonary disease & $42(8.5)$ \\
Extracardiac arteriopathy & $111(12.0)$ \\
Carotid artery stenosis & $68(7.3)$ \\
Lower limb ischemia & $60(6.5)$ \\
Hypertension & $369(39.8)$ \\
Hyperlipidemia & $289(31.1)$ \\
Diabetes & $135(14.5)$ \\
Transient ischemic attack/stroke & $46(5.0) / 46(5.0)$ \\
Type of operation & \\
$\quad$ Elective & $749(80.7)$ \\
Urgent & $174(18.8)$ \\
Emergency & $5(0.5)$ \\
Recent myocardial infarction $(>3 \mathrm{mo})$ & $92(9.9)$ \\
Atrial fibrillation & $51(5.5)$ \\
Prior cardiac operation & $14(1.5)$ \\
Ventricular septal rupture & $1(0.1)$ \\
Left ventricular ejection fraction $<50 \%$ & $128(13.8)$ \\
Left ventricular ejection fraction $<40 \%$ & $49(5.3)$ \\
Serum creatinine (mg/dL) & $93.3 \pm 32.6$ \\
\hline
\end{tabular}

Continuous variables are reported as the mean \pm SD.

At multivariate analysis, carotid artery disease $(P=.004)$, history of TIA $(P=.04)$, age $(P=.02)$, and diabetes $(P=.03)$ were independent predictors of postoperative stroke. The NNECVDSG stroke risk score had an area under the ROC curve of $0.758(P<.0001 ;$ SE, $0.060 ; 95 \%$ confidence interval $[\mathrm{CI}]$, 0.640-0.875) for prediction of postoperative stroke; its best cutoff value was 4 (Figure 1). Among patients with an NNECVDSG score of 4 or less, $0.8 \%$ had a postoperative stroke, whereas the stroke rate was $4.1 \%$ among those with a score greater than $4(P$ $=.001)$. The NNECVDSG stroke risk score had an area under the curve in predicting postoperative stroke/TIA of $0.759(P<.0001$; SE, 0.054; 95\% CI, 0.653-0.864).

The McSPI stroke risk score had an area under the ROC curve of $0.793(P<.0001$; SE, $0.05 ; 95 \%$ CI, 0.686-0.900) for prediction of postoperative stroke, and its best cutoff value was 77 (Figure 1). Among patients with a McSPI score of 77 or less, $0.7 \%$ had a postoperative stroke, whereas the stroke rate was $7.0 \%$ among those with a score greater than $77(P<.001)$. The McSPI stroke risk score had an area under the ROC curve in predicting postoperative stroke and TIA of $0.794(P<.0001$; SE, $0.050 ; 95 \% \mathrm{CI}$, 0.696-0.864). 

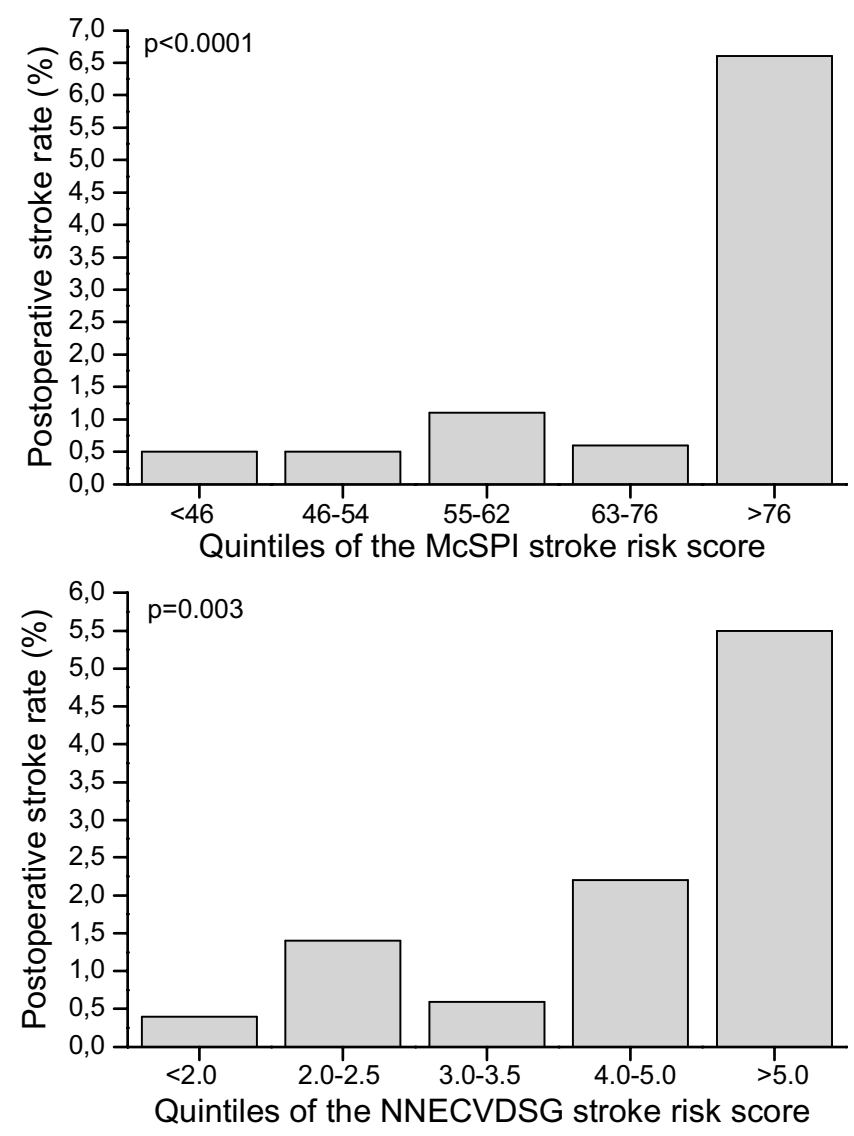

Figure 1. Quintiles of Northern New England Cardiovascular Disease Study Group (NNECVDSG) and Multicenter Study of Perioperative Ischemia (McSPI) stroke risk scores and the related postoperative stroke rates.

At 12 years, overall survival was $69.3 \%$, survival freedom from fatal cardiac events was $84.0 \%$, survival freedom from fatal stroke was $97.4 \%$ (273 deaths; 20 had fatal strokes; 336 patients entered this interval), and survival freedom from fatal cerebrovascular events was $96.3 \%$.
Diabetes $(P=.002$; relative risk [RR], 6.814; 95\% CI, 1.96923.581) and age ( $P=.02$; RR, 1.120; 95\% CI, 1.018-1.232) were independent predictors of fatal late stroke. The 12-year survival freedom from fatal stroke was $98.6 \%$ in patients with an NNECVDSG stroke risk score of 4 or less, whereas it was $94.6 \%$ in those with an NNECVDSG stroke risk score greater than $4(P=$ .0002). The 12-year survival freedom from fatal stroke was $98.2 \%$ in patients with a McSPI score of 77 or less, whereas it was $93.8 \%$ in those with a McSPI score greater than $77(P=.0007$; Table 1$)$.

\section{Discussion}

This study confirmed the value of these risk-scoring methods in predicting the risk of postoperative stroke in patients undergoing on-pump CABG. This finding has major research implications because it enables the identification of very-high-risk patients on which any neuroprotection method or drug should be evaluated. Furthermore, it enables clinicians to estimate before surgery and reduce during surgery the risk of such a severe complication by using the currently available methods. This would practically lead to identification of those patients at an extremely high risk of immediate postoperative stroke, as well as late fatal stroke. These patients are likely the ones who would benefit most from more extensive preoperative investigation of the carotid arteries, intraoperative epiaortic ultrasound examination of the ascending aorta, and, possibly, routine postoperative prophylactic anticoagulation. In particular, investigation of the carotid arteries in high-risk patients could be of importance in reducing the risk of any late disabling or fatal stroke by aggressive secondary prevention policy, as well as carotid endarterectomy, when appropriate.

\section{References}

1. Charlesworth DC, Likosky DS, Marrin CAS, Maloney CT, Quinton $\mathrm{HB}$, Morton JR, et al. Development and validation of a prediction model for stroke after coronary artery bypass surgery. Ann Thorac Surg. 2003;76:436-43.

2. Newman MF, Wolman R, Kanchurger M, Marschall K, Mora-Mangano C, Roach G, et al. Multicenter preoperative stroke risk index for patients undergoing coronary artery bypass graft surgery. Circulation. 1996; 94(suppl 2):II74-80.

3. Vuorisalo S. Surgical site infections after coronary artery bypass surgery. With special reference to antibiotic prophylaxis and risk factors [thesis]. Oulu: Acta Universitatis Ouluensis Medica, D 428, 1997. 